\title{
Light-induced relief gratings and a mechanism of metastable light-induced expansion in chalcogenide glasses
}

\author{
S. H. Messaddeq, ${ }^{1} *$ V. K. Tikhomirov, ${ }^{2}$ Y. Messaddeq, ${ }^{3}$ D. Lezal,,${ }^{4}$ and M. Siu $\mathrm{Li}^{1}$ \\ ${ }_{1}^{1}$ Instituto de Física de São Carlos-Universidade de São Paulo, C.P. 369, CEP: 13560-970, São Carlos, SP, Brazil \\ ${ }^{2}$ Mechanical Materials Manufacturing Engineering \& Management, University of Nottingham, Nottingham NG7 2RD, United Kingdom \\ ${ }^{3}$ Instituto de Química-UNESP-C.P. 355, CEP: 14801-970, Araraquara, SP, Brazil \\ ${ }^{4}$ Laboratory of Inorganic Materials IIC ASCR and ICT, Pelleova 24, Prague 6, Czech Republic \\ (Received 14 April 2000; revised manuscript received 23 January 2001; published 21 May 2001)
}

\begin{abstract}
We report on a metastable light-induced volume expansion in $\mathrm{Ge}_{25+x} \mathrm{Ga}_{10-x} \mathrm{~S}_{65}$ glasses under irradiation with band gap (UV) light, which can result in recording of relief gratings on their surface in the case of irradiation with two interfering beams. We propose a mechanism for the expansion, which is based on the light-induced change in the polarizability of secondary (van der Waals type) bonds and the effect of this change on primary (covalent type) bonds of the glass. The effect is suggested to be due to an interference of electrons, which belong to a chalcogen atom and participate in the formation of secondary and primary bonds, respectively. We suggest that a minimum point of the Lennard-Jones potential, which corresponds to the equilibrium position of a chalcogen atom is shifted in the course of irradiation to a larger interatomic distance. This shift causes a volume expansion and allows a diffusion of chalcogen atoms into the irradiated area. We show that light-induced polymerization of the glass network is an important attribute of the light-induced volume expansion.

DOI: 10.1103/PhysRevB.63.224203

PACS number(s): 61.43.Fs, 42.79.Dj, 42.70.Gi, 42.70.Ce
\end{abstract}

\section{INTRODUCTION}

Chalcogenide, especially sulfide and selenide, glasses show a metastable volume expansion (in some cases a contraction) in the course of irradiation by the visible or UV light. ${ }^{1-5}$ Recently it was shown that in $\operatorname{arsenic}^{2}$ and in germanium $^{3,4}$ sulfide glasses and in glassy and crystalline selenium, ${ }^{5}$ the magnitude of the effect can be much larger if a micron-size laser beam ${ }^{2,3,5}$ or two interfering beams ${ }^{4}$ are used for irradiation of the sample. The metastable volume expansion profile reproduces the transversal distribution of the intensity of the inducing single beam or of the spatial patterns of two interfering beams. Consequently, microlenses ${ }^{2}$ or profile gratings ${ }^{4}$ can be fabricated on the surface of the sample without any intermediate steps, such as etching. In addition, the effect of the expansion is thermally reversible; ${ }^{1}$ i.e., it can be removed after annealing of the sample near to the glass transition temperature. A thermally irreversible component of the metastable photoexpansion was also reported. ${ }^{4}$ It is similar to the thermally irreversible component of photodarkening or photobleaching, ${ }^{6}$ which usually accompany ${ }^{1}$ the metastable light-induced expansion.

The previous paper ${ }^{4}$ dealt with experimental characterization of the metastable light-induced volume expansion in $\mathrm{Ge}-$ $\mathrm{Ga}-\mathrm{S}$ and Ge-Ga-As-S glasses such as reciprocity low between the power density of irradiation and exposure time. In this paper, we aim at the elucidation of the mechanism for the metastable expansion in these glasses. We suggest that secondary chemical bonds with a high polarizability, such as van der Waals bonds, and the light-induced change in their polarizability are responsible for the effect of the metastable expansion. An interaction of these bonds with primary strong (covalent) bonds is suggested to determine the magnitude of the metastable volume change. The interaction is suggested to be due to an interference of electrons, which form primary and secondary chemical bonds, respectively; similar to an interference of electrons that was discussed in Ref. 7. We discuss the origin of high diffusivity of sulfur in the irradiated area based on the consideration of the Lennard-Jones potential for the interaction of sulfur with surrounding atoms, which changes in the course of irradiation. Similar considerations may be applicable for the metastable light-induced volume expansion, which is observed in selenides, and in some polymer films. ${ }^{8}$ In polymers, the light-induced change in the polarizability of hyperpolarizable $\pi$ bonds can be responsible for the effect.

\section{EXPERIMENT}

Glass samples were prepared by melting the high purity elemental compounds ( $\mathrm{Ge}, \mathrm{Ga}, \mathrm{As}$, and $\mathrm{S}$ ) in quartz ampoules evacuated up to $10^{-3} \mathrm{~Pa}$ and sealed with a torch. A distillation of compounds was carried out in a closed system. Following $6 \mathrm{~h}$ melting at $900^{\circ} \mathrm{C}$, the ampoule was quenched in water and annealed at $400{ }^{\circ} \mathrm{C}$, which is near to the glass transition temperature. Glass rods about $60 \mathrm{~mm}$ length and $10 \mathrm{~mm}$ diameter were obtained after cutting the ampoules. $\mathrm{X}$-ray analysis and scanning electron microscopy confirmed vitreous character and homogeneity of samples. $2 \mathrm{~mm}$ thick samples were cut and polished to the optical quality.

A modification with gallium is used to improve the glass stability of germanium sulfide glasses. It is reported that the $\mathrm{Ge}_{25} \mathrm{Ga}_{5} \mathrm{~S}_{65}$ composition lies in the center of the glassforming region in the ternary system Ge-Ga-S. ${ }^{9}$ On the other hand, we have observed a decrease of the amplitude of lightinduced relief gratings with varying the glass composition around $\mathrm{Ge}_{25} \mathrm{Ga}_{5} \mathrm{~S}_{65}$ (e.g., with varying a $\mathrm{Ge} / \mathrm{Ga}$ ratio or with 


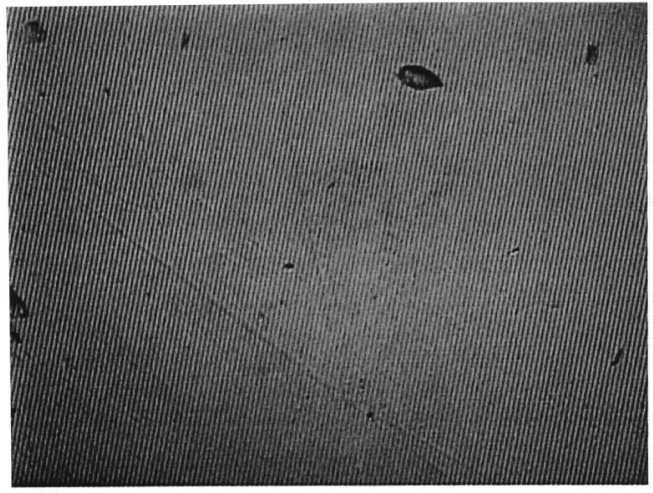

(a)

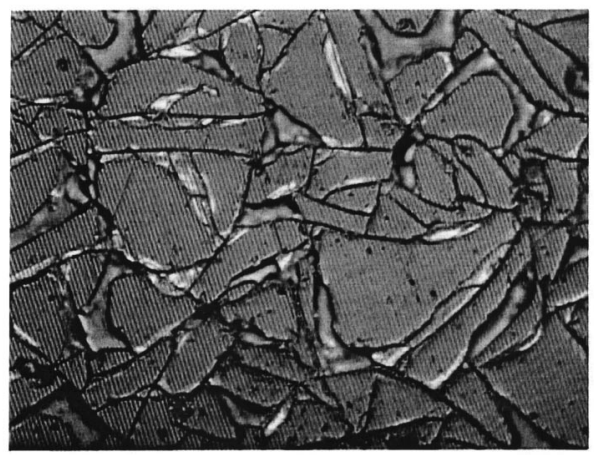

(b)

FIG. 1. Microphotographs of the surface of (a) $2 \mathrm{~mm}$ thick $\mathrm{Ge}_{25} \mathrm{Ga}_{10} \mathrm{~S}_{65}$ glass sample in the area (a) irradiated by two interfering beams of a cw $\mathrm{Ar}^{+}$laser $\left(\lambda=351 \mathrm{~nm}, P=0.75 \mathrm{~W} / \mathrm{cm}^{2}\right.$ each $)$ with an interference angle between beams equal to $3^{\circ}$. Pattern spacing is equal to 1.5 micron; the depth of relieves is equal to 0.19 micron. (b) In the same area after annealing for $20 \mathrm{~min}$ at $380{ }^{\circ} \mathrm{C}$.

an addition of As). Therefore we carried out experiments mostly on $\mathrm{Ge}_{25} \mathrm{Ga}_{5} \mathrm{~S}_{65}$ glasses.

A cw $\mathrm{Ar}^{+}$laser beam $\left(\lambda=351 \mathrm{~nm}\right.$, up to $\left.15 \mathrm{~W} / \mathrm{cm}^{2}\right)$ was used for recording the gratings. The laser beam was divided in two beams of equal intensities by means of splitter and mirror. These two beams were interfering on the glass surface. The angle between the beams was equal to $3^{\circ}$. A relief diffraction grating was recorded on the glass surface with fringes parallel to the surface. A profilometer was used for the direct measurement of the height of relieves. The kinetics of the diffraction efficiency were probed with a cw $\mathrm{He}-\mathrm{Ne}$ laser beam $(\lambda=633 \mathrm{~nm})$ incident orthogonal to the surface. The He-Ne laser beam could not affect the grating efficiency since an absorption coefficient of samples was negligible at $633 \mathrm{~nm}$.

The refractive index of samples was measured by an ellipsometer (SOPRA Spectroscopic Ellipsometer ESVG) in the range 300-900 $\mathrm{nm}$. The measurements were taken in the center of the irradiated spot on the sample surface.

\section{RESULTS}

A typical microphotograph of the recorded relief grating on the surface of the Ge-Ga-S glass is shown in Fig. 1(a). The microphotograph was taken after a completion of the

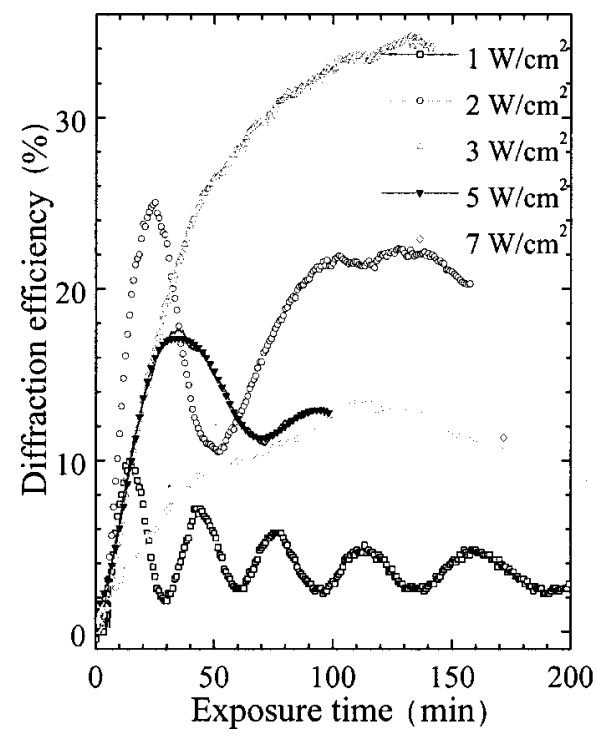

FIG. 2. The kinetics of the diffraction efficiency of relief gratings recorded in the same conditions as in Fig. 1(a) and probed with a cw He-Ne laser at $633 \mathrm{~nm}$. A power density of interfering UV beams is indicated in the inset.

photoinduced increase in the relieves height as it was confirmed by a stabilization of the diffraction efficiency. A direct measurement with a profilometer showed that after a completion of the photoinduced increase in the relieves height the maxima of relieves were 0.095 micron above and minima 0.095 micron below the unirradiated part of the surface. Hence the total depth of the relief grating was 0.19 micron. When this grating was given to the heat treatment for $20 \mathrm{~min}$ at $380^{\circ} \mathrm{C}$, i.e., $20^{\circ} \mathrm{C}$ below the glass transition temperature, the cracks on the surface appeared as it is seen in Fig. 1(b). A further heat treatment for longer than $1 \mathrm{~h}$ resulted in the disappearance of the cracks and a restoration of the flat surface as it was confirmed using a profilometer. The procedure for inscription and annealing of the relief gratings was repeated many times without a decrease in the amplitude of the volume expansion. This means that the metastable light-induced expansion is a thermally reversible effect, similar to the thermally reversible photodarkening/photobleaching. ${ }^{6}$

Figure 2 shows the kinetics of the diffraction efficiency in the same sample as in Fig. 1. The kinetics was probed with a $\mathrm{cw} \mathrm{He}-\mathrm{Ne}$ laser at $633 \mathrm{~nm}$. It is seen that the optimum power density was equal to $3 \mathrm{~W} / \mathrm{cm}^{2}$ when the kinetics was monotonous and the diffraction efficiency reached the largest value. The kinetics was nonmonotonous and sometimes even oscillating at lower and higher power density of interfering beams.

We have examined the morphology of the irradiated glass surface. In the case presented in Fig. 1, the EDX analysis indicated an increase of the sulfur content in the maxima of relief gratings up to $80 \%$ by atomic weight, while the amount of sulfur in the minima was decreased down to 50\%. An irradiation did not affect the $\mathrm{Ge} / \mathrm{Ga}$ ratio indicating that $\mathrm{Ge}$ and $\mathrm{Ga}$ atoms did not diffuse.

We have measured metastable light-induced changes in 


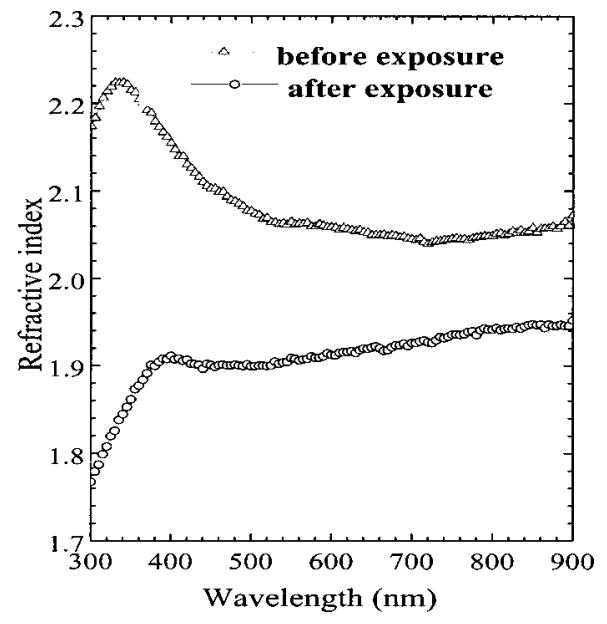

FIG. 3. The dispersion of the refractive index in the $\mathrm{Ge}_{25} \mathrm{Ga}_{10} \mathrm{~S}_{65}$ glass sample before (triangles) and after (circles) a 30 min exposure to the single $\mathrm{cw} \mathrm{Ar}{ }^{+}$laser beam at $P=1.5 \mathrm{~W} / \mathrm{cm}^{2}, \lambda=351 \mathrm{~nm}$.

the absorption coefficient and refractive index that usually accompany the light-induced expansion in chalcogenides. We found a shift of the absorption edge to the shorter wavelengths (photobleaching) after irradiation of our samples. We observed oscillations of the transmission at large power densities similar to that which was detected in $\mathrm{GeS}_{2}$ glasses in Ref. 10. We found a negative change of the refractive index in the irradiated area. The oscillations of the absorption coefficient and the respective oscillations of the refractive index can be responsible for the oscillations of the diffraction efficiency seen in Fig. 2. The value of the diffraction efficiency in Fig. 2 is determined by contributions from the space modulation of the surface relief and refractive index.

Figure 3 shows a dispersion of the refractive index in the $\mathrm{Ge}-\mathrm{Ga}-\mathrm{S}$ glass sample before and after exposure to the inducing $\mathrm{cw} \mathrm{Ar}^{+}$laser beam. The dispersion curve before exposure of the sample had a pronounced maximum of the refractive index at $330 \mathrm{~nm}$. A change of the dispersion curve was observed after irradiation: the maximum became weak and shifted to $400 \mathrm{~nm}$ while a subtle increase of the refractive index with wavelength was detected in the red part of the spectrum. The shift of the maximum to longer wavelengths and its weakening perhaps indicates that, in addition to the shift of the absorption edge to the shorter wavelengths, the edge also became less steep (shallower). The increase of the refractive index with wavelength in the red and infrared parts of the spectrum (anomalous dispersion) seems to be surprising however this result was reproductive. We guess that this anomalous dispersion is due to unhomogeneity of the glass structure after an exposure, which can be expected due to a diffusion of sulfur and a respective phase separation. It is known that the sulfur deficient phase $\left(\mathrm{Ge}_{50} \mathrm{~S}_{50}\right.$, rock salt structure) is a metastable phase and it has a crystalline counterpart. ${ }^{11}$ The electronic absorption edge of $\mathrm{Ge}_{50} \mathrm{~S}_{50}$ glass (which is of black color) is well shifted to the red, and even to the infrared, as compared to the electronic absorption edge of sulfur enriched germanium gallium sulfide glasses (which are of yellow color). ${ }^{11}$ The relationships, such as Kramers-Kronig and Wemple-Di Domenico, are not appli- cable to the case of inhomogeneous medium consisting of phases with different electronic absorption edges because the position of the absorption resonance is not uniquely defined. The presence of the $\mathrm{Ge}_{50} \mathrm{~S}_{50}$ phase (a partial phase separation) can explain anomalous dispersion in Fig. 3. EDX measurement (mentioned above) confirms the presence of $\mathrm{Ge}_{50} \mathrm{~S}_{50}$ phase in the irradiated area. In our opinion, the anomalous dispersion in Fig. 3 confirms the light stimulated diffusion of sulfur.

\section{DISCUSSION}

We attempt to give a qualitative explanation to the lightinduced volume expansion based on a specific anisotropic chemical bonding in chalcogenides. Chemical bonds in chalcogenides comprise of strong intramolecular covalent bonds (primary bonds which are due to binding $s-p$ hybridized electrons of chalcogen atom) and weak intermolecular van der Waals type bonds (secondary bonds which are due to lone-pair $p$-electrons of chalcogen atom), see, e.g., Refs. 7 and 12. (This is except for Te-based chalcogenides where bonds are mostly metallic ${ }^{7}$ and perhaps therefore no metastable light-induced volume expansion has been reported in them.)

An interatomic potential, which we assume is a LennardJones potential (see, e.g., Ref. 13), for interaction of sulfur with surrounding atoms can be written as follows:

$$
\Delta U=\frac{B}{R^{12}}-\frac{A\left(\alpha^{2}\right)}{R^{6}} .
$$

A minimum point of this potential corresponds to a balance between an attraction van der Waals type force $\left(-A / R^{6}\right.$ term) and a repulsion force related to an overlap of electronic wave functions $\left(B / R^{12}\right.$ term). Parameter $A$ is proportional to the square of the electronic polarizability $\alpha,{ }^{13}$ what is basically used in our approach. This potential is rather anisotropic in chalcogenides because both primary and secondary bonds contribute to it. Polarizability of weak secondary bonds $\alpha_{s}$ is much larger than polarizability of strong primary bonds $\alpha_{p}:\left(\alpha_{s} \gg \alpha_{p}\right)$, therefore the light-induced change of $\alpha_{s}$ will be larger then the change of $\alpha_{p}\left(\Delta \alpha_{s} \gg \Delta \alpha_{p}\right)$.

Since a remarkably large negative photorefraction $(\Delta n$ $\approx-0.2$ ) is detected in our experiments, see Fig. 3 , an averaged electronic polarizability (which is mostly due to $\alpha_{s}$, since $\alpha_{s} \gg \alpha_{p}$ ) decreases with irradiation as the mechanism of photorefraction is primarily related to the light-induced change of the electronic polarizability. ${ }^{14}$ Whereby an attraction term in Eq. (1) becomes weaker and first of all it becomes weaker in the directions where it is due to weak secondary bonds. A minimum point of the Lennard-Jones potential shifts with irradiation to a larger distance between sulfur and its ligands to which it is linked by secondary bonds. Since the similar process occurs in the environment of all sulfur atoms, the metastable expansion of the glass volume proceeds on a macroscopic scale.

We suggest that a magnitude of the expansion is restricted by the strength of the interaction (degree of interference ${ }^{7}$ ) between $s-p$ hybridized and $p$-electrons participating in the 
primary and secondary bonds, respectively. Specifically, in the case of our Ge-Ga-S glasses, light-induced weakening in the secondary bonds should result in strengthening of the primary bonds as it is argued in Ref. 7 whereby limiting a magnitude of the glass network expansion. The symmetry and parity of electronic clouds participating in secondary and primary bonds centred at the chalcogen atom have a primary effect on the character of their interaction via interference. The degree of $s-p$ hybridization, which is determined by a specific structure of the glass network (namely by a structure of sulfur sites) is to determine the magnitude and the sign of the volume change and photorefraction.

Contrary to Ge-Ga-S glasses, a metastable light-induced expansion with small positive photorefraction $(\Delta n$ $=+0.01$ ) is reported in the $\mathrm{As}_{2} \mathrm{~S}_{3}$ glass (see Ref. 2 and references therein). Therefore, we are to assume light-induced strengthening of the secondary bonds in the case. ${ }^{2}$ The reason for this contrary behavior can be in the difference of sulfur sites in germanium and arsenic sulfide glasses resulting in a different character of $s-p$ hybridization. (While the structure of $\mathrm{As}_{2} \mathrm{~S}_{3}$ glass is dominated by As-S-As-S rings with bridging and nonbridging sulfur atoms, ${ }^{12,15}$ the structure of stoichiometric germanium sulfide glasses is dominated by $\mathrm{GeS}_{1}$ tetrahedra (see, e.g., Ref. 16 and refs therein) and the structure of Ge-enriched germanium sulfide glasses is dominated by Ge-S-Ge-S chains. ${ }^{11,16}$ Strengthening of secondary bonds should result in weakening of the primary bonds ${ }^{7}$ what can result in the volume expansion. ${ }^{2}$ Noteworthy, the nonmonotonous kinetics of the light-induced volume expansion (an expansion at the first stage and then a contraction) was observed in $\mathrm{As}_{2} \mathrm{~S}_{3}$ films. ${ }^{17}$ The nonmonotonous kinetics of the expansion at some power densities of the light is also evidenced in some of our experiments; see Fig. 2. A nonmonotonous character of the light-induced volume change can be due to instability of a balance between primary and secondary bonds that may alter in the course of irradiation. A shallow well (or even presence of two wells) in the LennardJones potential (1) is a prerequisite for the nonmonotonous behavior of the expansion.

Regarding our experimental data about enrichment with sulfur in the maxima of relieves, we note that weakening of secondary bonds centered at sulfur atom can result in the movement of sulfur atom to the free volume, which appears in its environment in the course of the light-induced expansion of the irradiated area. Furthermore, the diffusion of sulfur into the irradiated area from the unirradiated surrounding can be a self-accelerating process since an enrichment in sulfur results in the extra secondary bonds and consequently in an accelerated light-induced expansion of the glass network. The micron scale size of the irradiated area (as mentioned in the Introduction, see Refs. 2-5) is an important aspect of the expansion process, because no efficient diffusion of sulfur from an unirradiated area into an irradiated area may proceed on the larger scale. Therefore the relative volume expansion of chalcogenides is much smaller when it is induced by millimeter scale beams. ${ }^{1,2}$

Photoinduced anisotropy is observed in sulfide (including Ge-Ga-S) and selenide glasses when irradiated by polarized or unpolarized light; see e.g., Ref. 18. This effect is based on the presence of two metastable states in the glass structure, which differ by anisotropy and chirality. ${ }^{18,19}$ Light-induced tunnelling of an over-coordinated photoexcited chalcogen atom was proposed to be in the origin of photoinduced anisotropy. The similar motion can be responsible for the diffusion of sulfur in our model for the metastable light-induced expansion where an over-coordination of the chalcogen atom is due to the presence of secondary bonds of van der Waals type (normal coordination of the chalcogen atom is equal to 2 and it is determined by the number of strong covalent bonds; see, e.g., Refs. 7 and 12). We suggest that photoexcited sulfur atoms will diffuse with high probability into voids, which appear due to the expansion of glass network in the vicinity of low-coordinated sulfur atoms. This will result in the creation of new S-S bonds (or in other words, in a polymerization of the glass network) in the irradiated area. Such a polymerization explains (see, e.g., Ref. 6) the effect of photobleaching, which accompanies a glass network expansion in the case of our glasses.

An importance of light-induced polymerization of glass network in the effect of light-induced expansion is indicated by the fact that the largest expansion was observed in the $\mathrm{Ge}_{25} \mathrm{Ga}_{5} \mathrm{~S}_{65}$ glass. An enrichment of this compound in sulfur (like in the ridges of photoinduced relieves) or germanium (like in the valleys of photoinduced relieves) results in polymerization of glass network [creation of S-S-S (Ref. 16) and Ge-S-Ge-S (Refs. 11 and 16) chains, respectively] and suppression of the light-induced expansion. A substitution of $\mathrm{Ge}$ for As also results in polymerization of glass network (creation of S-As-S chains) due to lower coordination number of $\mathrm{As}(3)$ as compared with $\mathrm{Ge}(4)$. Finally, $\mathrm{Ge}_{x} \mathrm{Ga}_{y} \mathrm{~S}_{65}$ compound with $x+y=35$ is a least polymerized compound amongst Ge-Ga-S glasses due to the presence of "ethanlike" $\mathrm{S}_{3}$-Ge-Ga- $\mathrm{S}_{3}$ units. ${ }^{20}$ Therefore we conclude that a small degree of the glass network polymerization is a prerequisite for its efficient light-induced expansion, which is accompanied (and maybe is due to) light-induced polymerization of the glass network.

Light-induced polymerization of chalcogenide glasses is known to be thermally reversible, ${ }^{6,21}$ therefore the lightinduced expansion is also thermally reversible, in agreement with our data ${ }^{4}$. We suggest that the thermal reversibility can be due to similar binding energy of S-S $[50.9 \mathrm{kcal} / \mathrm{mol}$ (Ref. 20)], Ge-S [51.7 kcal/mol (Refs. 20 and 22)], and Ga-S [55.2 $\mathrm{kcal} / \mathrm{mol}$ (Ref. 22)] bonds, where binding energy is calculated based on values of single bond strength and electronegativity of respective atoms. ${ }^{23}$

\section{CONCLUSION}

We presented experimental data on the new effect: lightinduced relief gratings on the surface of $\mathrm{Ge}-\mathrm{Ga}-\mathrm{S}$ bulk glasses produced by an interference of two UV laser beams. We suggested the microscopic mechanism for this effect based on the interference of the $p$ and $s-p$ hybridized electrons centered at the chalcogen atom and participating in the formation of weak van der Waals type (secondary) and strong covalent (primary) bonds, respectively. The diffusion 
of chalcogen atoms into the irradiated area is observed and explained as due to weakening of the bonds centered at the chalcogen atom. Based on our experimental data on the varying the magnitude of the volume expansion with the glass composition, we emphasized an importance of light-induced glass network polymerization in the effect of the volume expansion.

*Corresponding author.

${ }^{1}$ H. Hamanaka, Ka. Tanaka, A. Matsuda, and S. Iizima, Solid State Commun. 19, 499 (1976); 23, 63 (1977).

${ }^{2}$ H. Hisakuni and Ke. Tanaka, Appl. Phys. Lett. 65, 2925 (1994); Opt. Lett. 20, 958 (1995); Ke. Tanaka, Phys. Rev. B 57, 5163 (1998).

${ }^{3}$ P. Loeffler, T. Schwarz, H. Sautter, and D. Lezal, J. Non-Cryst. Solids 232\&234, 526 (1998).

${ }^{4}$ S. H. Messaddeq, M. Sui Li, Y. Messaddeq, D. Lezal, and S. J. L. Ribeiro, J. Non-Cryst. Solids (to be published).

${ }^{5}$ V. V. Poborchii, A. V. Kolobov, and Ka. Tanaka, Appl. Phys. Lett. 74, 215 (1999).

${ }^{6}$ J. P. De Neufville, S. C. Moss, and S. R. Ovshinsky, J. NonCryst. Solids 13, 191 (1973).

${ }^{7}$ R. M. Martin, G. Lucovsky, and K. Helliwell, Phys. Rev. B 13, 1383 (1976).

${ }^{8}$ G. Cipparone, A. Mazzulla, F. P. Nicoletta, and F. Simoni, Opt. Commun. 150, 297 (1998).

${ }^{9}$ F. Gan, J. Non-Cryst. Solids 123, 385 (1990); Y. Guimond et al., ibid. 256\&257, 378 (1999).

${ }^{10}$ V. M. Lyubin and V. K. Tikhomirov, J. Non-Cryst. Solids 164\&166, 1211 (1993).

${ }^{11}$ Y. Kawamoto and S. Tsuchihashi, J. Am. Ceram. Soc. 54, 131 (1971).

${ }^{12}$ R. Zallen, M. L. Slade, and A. T. Ward, Phys. Rev. B 3, 4257 (1971).

\section{ACKNOWLEDGMENTS}

V.K.T. gratefully acknowledges Professor H. Fritzsche for the stimulated discussion about chemical bonds in chalcogenides. The authors would like to thank Professor M. A. Aegerter and Dr. P. Oliveira (Institut fur Neue Materialen, Saarbrucken, Germany) for very fruitful discussion.

${ }^{13}$ C. Kittel, Introduction to Solid State Physics, 7th ed. (Wiley, New York, 1996), p. 56.

${ }^{14} \mathrm{P}$. Yeh, Introduction to Photorefractive Nonlinear Optics (Wiley, New York, Chichester, 1993).

${ }^{15}$ J. P. Mathieu and H. Poulet, Bull. Soc. Fr. Mineral. Cristallogr. 93, 532 (1970); R. J. Kobliska and S. A. Solin, Phys. Rev. B 8, 756 (1973); R. Zallen and M. L. Slade, ibid. 9, 1627 (1974); G. Lucovsky and R. M. Martin, J. Non-Cryst. Solids 8\&10, 185 (1972).

${ }^{16}$ P. Boolchand, J. Grothaus, M. Tenhover, M. A. Hazzle, and R. K. Grasselli, Phys. Rev. B 33, 5421 (1986).

${ }^{17}$ A. Ganjoo, Y. Ikeda, and K. Shimakawa, J. Non-Cryst. Solids 266\&269, 919 (2000).

${ }^{18}$ V. M. Lyubin and V. K. Tikhomirov, J. Non-Cryst. Solids 114, 133 (1989); V. K. Tikhomirov and S. R. Elliott, Phys. Rev. B 51, 5538 (1995); H. Fritzsche, J. Non-Cryst. Solids 164\&166, 1169 (1993).

${ }^{19}$ V. K. Tikhomirov, J. Non-Cryst. Solids 256\&257, 328 (1999); V. K. Tikhomirov, G. J. Adriaenssens, and S. R. Elliott, Phys. Rev. B 55, R660 (1997).

${ }^{20} \mathrm{P}$. Boolchand et al. (private communication) (work in progress).

${ }^{21}$ S. R. Elliott, J. Non-Cryst. Solids 81, 71 (1986); G. Pfeiffer, M. A. Paesler, and S. C. Agarwal, ibid. 130, 111 (1991).

${ }^{22}$ J. Sanghera (private communication) (estimation based on values of single bond strength and electronegativity).

${ }^{23}$ L. Pauling, Nature of the Chemical Bond (Cornell University Press, Ithaca, 1960), p. 85. 